\title{
COMPOSIÇÃO DE MACROINVERTEBRADOS BENTÔNICOS DO CÓRREGO BELA VISTA, ARAÇATUBA-SP
}

\author{
Ricardo Haruo Nakamura Kanno \\ Germano Henrique Costa Barrili \\ Natalia Felix Negreiros
}

RESUMO; A comunidade de macroinvertebrados bentônicos tem sido amplamente utilizada por apresentar grande diversidade de organismos, com diferentes graus de tolerância à poluição, por possuir pouca mobilidade e por possuir um ciclo de vida relativamente longo. A comunidade de macroinvertebrados bentônicos é representada principalmente pela Classe Insecta (Diptera, Ephemeroptera, Odonata, Hemiptera e Coleoptera) e pelos Filos Platyhelminthes, Nematoda, Anellida e Mollusca. No presente estudo, estes organismos foram utilizados como bioindicadores da qualidade da água do Córrego Bela Vista, Araçatuba-SP. Foram realizadas 3 coletas em 3 pontos de amostragem. Para a captura dos organismos foi utilizado um amostrador tipo Surber, e determinadas, in situ e em laboratório, as variáveis físicas e químicas da água e do sedimento. Os organismos foram identificados até o menor nível taxonômico possível, utilizando-se as chaves e guias de identificação específica. Na primeira coleta foram encontrados 770 organismos, divididos entre os táxons Chironomidae, Oligochaeta, Nematoda, Hydracarina, Hirudinea e Tabanidae, Na segunda coleta foram encontrados 25 organismos divididos em Chironomidae, Oligochaeta, Hydrophilidae e Hydrobiidae. $\mathrm{Na}$ terceira coleta foram encontrados 491 organismos divididos entre os grupos Chironomidae, Oligochaeta, Hydrophilidae, Elmidae, Gerridae e Cossidae. Os indivíduos da família Chironomidae estiveram presentes em todas as amostragens e representaram mais da metade de todos os organismos encontrados, comprovando que estes indivíduos são adaptados a quase todos os ecossistemas aquáticos e semiaquáticos.

Palavras chave: Chironomidae; Oligochaeta; Córrego Belo Vista Araçatuba-SP 


\section{INTRODUÇÃO}

Alterações antrópicas em corpos hídricos são consequências indiretas do aumento populacional, que acabam por elevar a necessidade de consumo dos recursos naturais, sejam para aquisição de matéria-prima, energia elétrica, solos mais férteis, entre outros. Através dessa utilização podem ocorrer a destruição de hábitats e a escassez de água potável. Além disso, o modelo atual de desenvolvimento e a política pública sobre o controle do crescimento populacional é ineficaz para a manutenção desses recursos naturais (BUSS, 2004). Outra atividade que afeta diretamente a qualidade da água é a utilização desenfreada dos solos (BOTELHO \& CAMPOS, 2001) que, assim como as atividades de mineração, despejos de efluentes, construção de barragens, mudanças no curso natural de rios, interferem na qualidade da água e na biodiversidade aquática ao alterarem as condições físicas, químicas e a dinâmica natural das comunidades biológicas encontradas nesses locais (GOULART e CALLISTO, 2003).

Um dos grandes problemas que afetam o país é a eutrofização artificial da água como consequência da intervenção do homem na natureza (GOULART e CALLISTO, 2003). As principais fontes de contaminação dos recursos hídricos são resíduos químicos, advindos das atividades industriais e pecuárias, além do despejo de rejeitos domésticos (EGLER, 2002).

A avaliação da poluição de um sistema aquático pode ser feita através de vários processos, dentre eles destaca-se a utilização de organismos indicadores. As técnicas de biomonitoramento são baseadas nos parâmetros de resposta dos organismos com relação ao ambiente em que vivem, já que a maioria dos ambientes está sujeita a alterações, seja nos processos físicos, químicos e/ou biológicos (KARR, 1991; SOUZA, 2001; BUSS et al., 2003).

A comunidade bentônica é composta por invertebrados, cujos tamanhos no final do estado larval ou na fase imaginal é raramente menor que um milímetro (TACHET et al., 1987). São encontrados nas regiões bentônicas (fundo) de ecossistemas aquáticos, como riachos, rios, represas e lagos, durante parte do seu ciclo ou toda vida, associado aos diversos tipos de substratos presentes. Esta comunidade tem sua distribuição controlada pela disponibilidade de alimento, tipo de sedimento/substrato, temperatura do meio, tipo 
de ecossistema, concentrações de oxigênio, $\mathrm{pH}$, presença de mata ciliar, além da competição entre as espécies. (WARD et al., 1995; ESTEVES. 1998; GALDEAN et al., 2000).

Os diversos grupos que coexistem dentro da comunidade dos macroinvertebrados bentônicos, apresentam diferentes níveis de tolerância em relação ao tipo de ambiente, e de acordo com Goulart \& Callisto (2003) e Moretti \& Moreno (2006) e estas características os classificam em três grupos: sensíveis ou intolerantes, organismos tolerantes e organismos resistentes. Os sensíveis, necessitarem de uma grande quantidade de oxigênio dissolvido na água, os principais representantes destes grupos são os Ephemeroptera, Trichoptera e Plecoptera. O grupo tolerante é formado por uma grande variedade de insetos aquáticos e outros invertebrados, principalmente das ordens Heteroptera, Odonata e Coleoptera, necessitam de menor concentração de oxigênio dissolvido. Já os organismos do grupo resistente são formados por espécies totalmente tolerantes, sendo capazes de viver em condição anóxia por várias horas. Este grupo é formado principalmente por larvas de Chironomidae e alguns Dipteras e por toda a classe das Oligochaetas (GOULART \& CALLISTO, 2003)

Os ecossistemas aquáticos dependem da proteção vegetal em suas margens para evitarem mudanças abruptas causadas por fenômenos naturais, além de contribuem para o equilíbrio ambiental (MONTAG et al., 1997; PASSOS, 1998; KAGEYAMA \& GANDARA, 1998; JOHNSON et al., 1999; LIMA \& ZAKIA, 2000). Dessa forma o objetivo do presente estudo foi o levantamento da comunidade de macroinvertebrados bentônicos no córrego Bela Vista que se encontra na bacia do Rio Tietê e na sub-bacia Baguaçu da cidade de Araçatuba interior de São Paulo.

\section{METODOLOGIA}

As coletas foram realizadas em Setembro de 2012, Janeiro e Maio de 2013 em uma área localizada no interior da Secretaria Municipal do Meio Ambiente e Sustentabilidade (SMMAS), na antiga fazenda do Estado, e em um trecho do córrego Bela Vista, no município de Araçatuba (SP) (Fig.1). 
Os dois primeiros pontos estão localizados na área do reflorestamento e o terceiro em uma região próxima à Rua Goulart, no bairro Santa Luzia, no município de Araçatuba$\mathrm{SP}$, sendo estes georeferenciados a seguir:

Ponto 1: $21^{\circ} 12.051^{\prime}$ S; $50^{\circ} 25.920^{\prime} \mathrm{W}$

Ponto 2: $21^{\circ} 12.084^{\prime}$ S; $50^{\circ} 25.829^{\prime} \mathrm{W}$

Ponto 3: e $21^{\circ} 12.076^{\prime}$ S; $50^{\circ} 25.634^{\prime} \mathrm{W}$
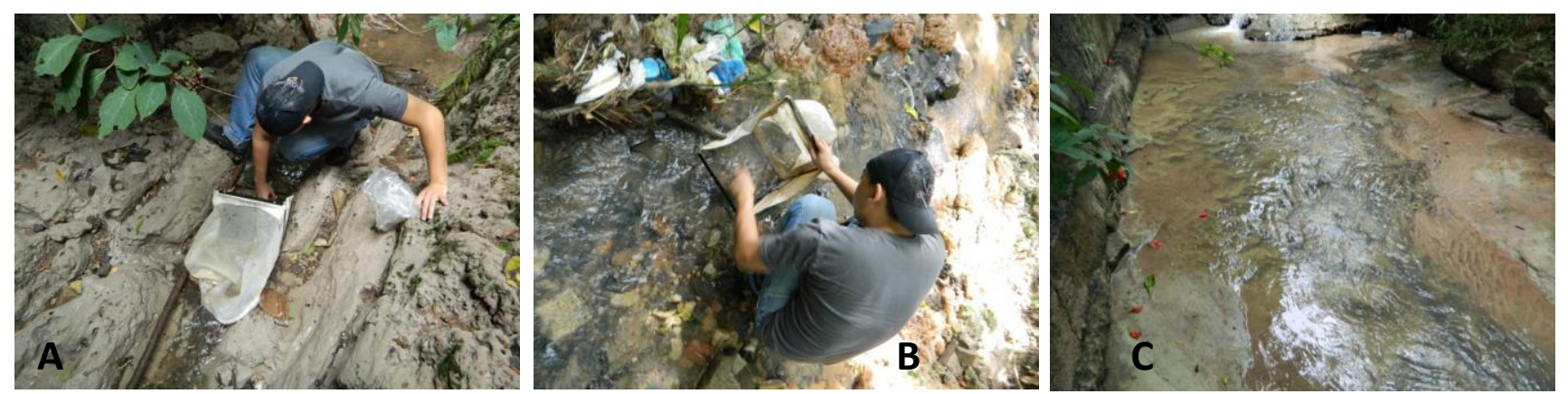

Figura 1. Localização dos pontos de amostragem. A - Ponto 1 (Interior da SMMAS); B Ponto 2 (Interior da SMMAS); C - Ponto 3, no município de Araçatuba-SP.

Em cada ponto de amostragem foram realizadas análises físicas e químicas da água e do sedimento coletado através dos métodos descritos por Golterman et al. (1978), Mackereth et al. (1978) e Valderrama (1981). As variáveis analisadas serão: concentração de oxigênio dissolvido, nitrogênio total (NT), fósforo total (PT), concentração de clorofila $a$.

Os organismos foram identificados utilizando-se as chaves e guias de identificação: Merritt e Cummins (1984); Pérez (1988); Epler (1992); Trivinho-Strixino \& Strixino (1995), Righi (1984) e Brinkhurst \& Marchese (1989), entre outras, e recorrendo-se ainda ao auxílio de especialistas.

A determinação da riqueza taxonômica (S) foi determinada pela contagem direta do número de táxons presentes nas amostras, e a diversidade de espécies (ShannonWierner - H') de cada ponto amostrado, calculada segundo Magurran (1988):

$$
\mathrm{H}^{\prime}=-\Sigma \mathrm{pi}{ }^{*} \log \mathrm{pi} \text { onde, }
$$

ni = número de indivíduos da espécie numa amostra;

$\mathrm{N}$ = número total de indivíduos presentes na amostra.

A equitabilidade será avaliada através da equação de Pielou (MAGURRAN, 1988): 


$$
\begin{aligned}
& \text { e = H' / Hmáx onde, } \\
& \text { e = equidade ou uniformidade da amostra,; } \\
& H^{\prime} \text { = diversidade de espécies observadas na amostra; }
\end{aligned}
$$

Para verificar a existência de correspondências entre os parâmetros ambientais e a densidade dos grupos da comunidade de macroinvertebrados bentônicos identificados no presente estudo foi realizada a Análise de Correspondência Canônica (CCA), através do software CANOCO 3.12 (TER BRAAK, 1986).

\section{RESULTADOS E DISCUSSÃo}

$\mathrm{Na}$ tabela 1 estão sumarizados os valores obtidos para as variáveis ambientais aferidos no Córrego Bela Vista, município de Araçatuba-SP, na primavera, verão e outono.

Tabela 1. Valores obtidos para as variáveis ambientais registradas nos três pontoss, localizados no Córrego Bela Vista, Araçatuba-SP. (Os traços - indicam ausência de aferição).

\begin{tabular}{cccccccccc}
\hline Variáveis & \multicolumn{3}{c}{ Primavera } & \multicolumn{3}{c}{ Verão } & \multicolumn{3}{c}{ Outono } \\
ambientais & P1 & P2 & P3 & P1 & P2 & P3 & P1 & P2 & P3 \\
NT & 5585,3 & 4769,9 & 4639,5 & - & - & - & - & - & - \\
PT & 235 & 212,8 & 40,2 & & & & & & \\
Cond. & 21 & 22 & 0,3 & 1.2 & 1.1 & 0,3 & 426 & 280 & 260 \\
OD & 8,3 & 8,5 & 8 & 8,9 & 12,5 & 12,2 & 2 & 2,9 & 6,7 \\
Temp & 24,6 & 24,2 & 20,3 & 28 & 24,5 & 24,9 & 23,7 & 23,8 & 22,6 \\
\hline
\end{tabular}

No total, foram encontrados 1477 organismos agrupados em 13 famílias (Chironomidae,Ooligochaeta,Nematoda, Tabanidae, Hirudínea, Hydrophilidae, Hydrobiidae, Gerridae, Cossidae, Empidida, Elmidae, hydrachnidae e Pupa). (Tabela 2).

Tabela 2. Número de indivíduos de macroinvertebrados bentônicos registrados nos três pontos das três coletas, localizados no Córrego Bela Vista, Araçatuba-SP.

\begin{tabular}{lccccccccccc}
\hline & \multicolumn{3}{c}{ Primavera } & \multicolumn{3}{c}{ Verão } & \multicolumn{3}{c}{ Outono } \\
\hline TAXÓNS & C1-P1 & C1-P2 & C1-P3 & C2-P1 & C2-P2 & C2-P3 & C3-P1 & C3-P2 & C3-P3 & TOTAL \\
\hline CHIRONOMIDAE & 23 & 364 & 383 & 3 & 4 & 11 & 91 & 30 & 325 & 1234 \\
\hline
\end{tabular}




\begin{tabular}{|c|c|c|c|c|c|c|c|c|c|}
\hline OLIGOCHAETA & 14 & 168 & - & 1 & 1 & 2 & - & - & 9 \\
\hline NEMATODA & 3 & 3 & 1 & - & - & - & - & - & - \\
\hline TABANIDAE & - & 2 & - & - & - & - & - & - & - \\
\hline HIRUDINEA & - & - & 2 & - & - & - & - & - & - \\
\hline HYDROPHILIDAE & - & - & - & - & 1 & - & 10 & 2 & 5 \\
\hline HYDROBIIDAE & - & - & - & - & - & 1 & - & - & - \\
\hline GERRIDAE & - & - & - & - & - & - & 1 & - & - \\
\hline COSSIDAE & - & - & - & - & - & - & 1 & - & - \\
\hline EMPIDIDAE & - & - & - & - & - & - & - & - & 1 \\
\hline ELMIDAE & - & - & - & - & - & - & 1 & - & - \\
\hline PUPA & - & - & 3 & - & 1 & - & 9 & - & - \\
\hline HIDRACHINIDAE & 1 & - & - & - & - & - & - & - & - \\
\hline TOTAL & 41 & 537 & 389 & & & & 113 & 32 & 340 \\
\hline
\end{tabular}

Como esperado os organismos mais abundantes foram os da família dos Chironomidae com 1234 dos organismos encontrados, representando mais de $83,5 \%$.

Tabela 3. Valores obtidos para a riqueza de táxons, número de indivíduos, índices de dominância (D'), Shannon-Wiener ( $\left.H^{\prime}\right)$ e equitabilidade ( $\left.\mathrm{J}^{\prime}\right)$ de macroinvertebrados bentônicos registrados nos três pontos das 3 coletas, localizados no Córrego Bela Vista, Araçatuba-SP.

$\begin{array}{lll}\text { COLETA } 1 & \text { COLETA2 } & \text { COLETA } 3\end{array}$

\begin{tabular}{lccccccccc}
\hline Indices & P1 & P2 & P3 & P1 & P2 & P3 & P1 & P2 & P3 \\
Riqueza & 4 & 4 & 4 & 2 & 4 & 3 & 6 & 2 & 4 \\
Individuos & 41 & 537 & 389 & 4 & 7 & 14 & 113 & 32 & 340 \\
D' & 0,4372 & 0,5574 & 0,9695 & 0,625 & 0,3878 & 0,6429 & 0,6629 & 0,8828 & 0,9146 \\
H' & 0,9731 & 0,6769 & 0,09525 & 0,5623 & 1,154 & 0,656 & 0,716 & 0,2338 & 0,2185 \\
J' & 0,7019 & 0,4883 & 0,06871 & 0,8113 & 0,8322 & 0,5971 & 0,3996 & 0,3373 & 0,1576
\end{tabular}


A porcentagem total explicada pelos dois primeiros componentes na análise correspondência de canônica (CCA) entre a densidade das famílias de macroinvertebrados bentônicos (Figura 1) e as variáveis ambientais foi de 86,0\%. As variáveis ambientais utilizadas na análise, segue na Tabela 3. As variáveis significativamente, evidenciadas pelo Teste de Monte Carlo foram: concentração de fósforo total e a concentração de oxigênio dissolvido.

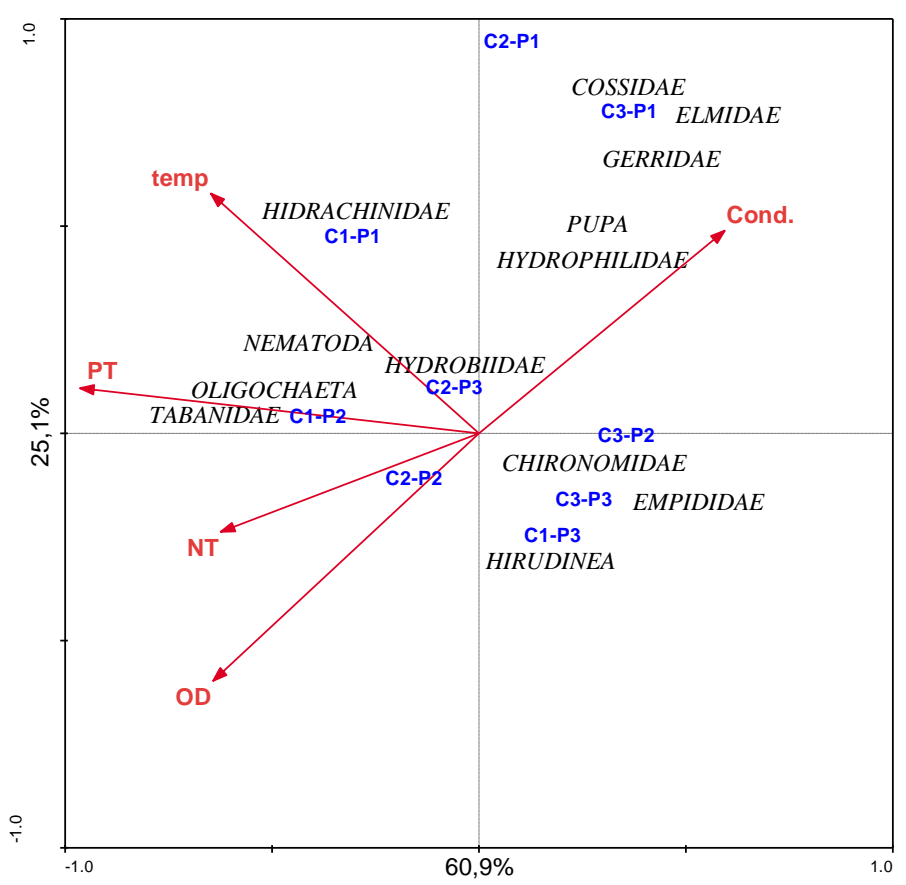

Figura 1: Diagrama de ordenação CCA com base nas densidades (org. $\mathrm{m}^{-3}$ ) das famílias e variáveis ambientais registrados nos três pontos das 3 coletas, localizados no Córrego Bela Vista, Araçatuba-SP.

No presente estudo a Família Chironomidae e os Oligochaetas foram os mais representativos numericamente. Estes dois grupos são possivelmente os mais adaptados em relação aos demais macroinvertebrados bentônicos, apresentando diversas adaptações ecológicas e biológicas que os tornam aptos a explorar diferentes hábitats devido a grande tolerância e resistência (Pinder, 1983). Vivem em todos tipos de águas doces, frequentemente atingindo elevadas densidades populacionais, podem ser indicadoras da qualidade ambiental, com algumas espécies possuindo exigências ambientais específicas e outras sendo relativamente tolerantes a condições adversas. 


\section{Then

Segundo Callisto et al. (2002), os Chironomidae são considerados os mais abundantes na comunidade bentônica e normalmente dominam os ecossistemas aquáticos devido à capacidade de tolerar condições extremas.

Com relação ao índice de Shannon-Wiener (Tabela 3), os dados dos táxons são combinados em um único valor que, supostamente, indica o estado da comunidade. Em geral esse índice passa a apresentar baixo valor em decresce diante de impactos ambientais (WASHINGTON, 1984).

De acordo com Wilhm e Dorris (1968) sugeriram como parâmetro: H' < 1,0 - forte poluição, $H^{\prime}$ = 1,0 - 3,0 poluição moderada e H' > 3,0 - água sem poluição. Diante disso, os três pontos amostrados podem ser considerados poluídos.

No ponto 3, ocorreu a maior dominância, consequência da superioridade numérica representada pelo grupo Chironomidae (Tabela 1). Este ponto está situado dentro da área urbana e também se trata da área mais impactada ocorrendo o despejo de lixo no córrego. Devido a essas condições, os únicos organismos encontrados são os com maior resistência a ambientes com muita poluição.

\section{CONCLUSÃO}

Em decorrência da retirada da mata ciliar, urbanização e atividades inerentes, o córrego Belo Vista se encontra negativamente impactado. Esses impactos refletiram no desaparecimento de grupos de macroinvertebrados bentônicos sensíveis e promoveram a dominância de espécies resistentes como os grupos Oligochaeta e Chironomidae

\section{REFERÊNCIAS}

ARAÇATUBA. Plano Municipal de Abastecimento de Água e Esgotamento Sanitário do Município de Araçatuba: Alternativas para o sistema de abastecimento de água e esgotamento sanitário com diretrizes de ampliação e melhoria dos sistemas - Versão Final. Relatório técnico - RT3. 2011. 158p. 
BUSS, D. F. Importância do Biomonitoramento e programas de analise de qualidade da agua dos rios. 2004. Disponível em: www.bioletin.hpg.ig.br/lll3/artigo/buss

BOTELHO, C. G.; CAMPOS, C. M. Recursos naturais e impacto ambiental. UFLA/FAEPE, 2001

BRINKHURST, R.O. \& MARCHESE, M.R. 1989. Guia para la indentificacion de Oligoquetos aquáticos continentales de Sud y Centroamerica. Climax, Santa Fé, Argentina. 207p.

EGLER, M. Utilizando a Comunidade de Macroinvertebrados Bentônicos na Avaliação da Degradação Ambiental de Ecossistemas de Rios em Áreas Agrícolas. RJ, Brasil. f. 147. 2002. Dissertação (Mestrado em Saúde Pública). Fundação Oswaldo Cruz. Rio de Janeiro, 2002.

EPLER, J.H. Identification manual for the larval Chironomidae (Diptera) of Florida. Orlando: Department of Environmental Protection, 1992.

ESTEVES, F. A. Fundamentos de limnologia. 2. ed. Rio de Janeiro: Interciência, 1998.

GALDEAN, N.; CALLISTO, M.; BARBOSA, F. A. R. Lotic Ecosystems of Serra do Cipó, southeast Brazil: water quality and a tentative classification based on the benthic macroinvertebrate community. Aquatic Ecosystem Health and Management. n. 3, p. 545-552, 2000.

GOLTERMAN, H.L.; CLYMO, R.S.; OHNSTAD, M.A.M. Methods for physical and chemical analysis of freshwaters. 2. ed. Oxford: Blackwell Scientific Publications, 1978, 213.

GOULART, M.; CALLISTO, M. Bioindicadores de agua como ferramenta em estudo de impacto ambiental. Revista FAPAM, 2003. 
JOHNSON, M. A.; SARAIVA, P. M.; COELHO, D. The role of gallery forests in the distribution of Cerrado mammals. Revista Brasileira de Biologia, v. 59, n. 3, 1999.

KAGEYAMA, P.; GANDARA, F. B. 1998. Consequências genéticas da fragmentação sobre populações de espécies arbóreas. Série Técnica IPEF, v. 12, n. 32, p. 65-70, 1998.

KARR, J. Biological integrity: a long neglected aspect of water resource management. Ecological Applications, v. 1, p. 26-35, 1991.

LIMA, W. P.; ZAKIA, M. J. B. Hidrologia de Matas Ciliares. In: RODRIGUES, R. R.; LEITÃO-FILHO, H. F. Matas Ciliares: conservação e recuperação. São Paulo: EDUSP/Editora da Universidade de São Paulo, 2000.

MACKERETH, J. F. H.; HERON, J. \& TALLING, J. F. Water analysis: some revised methods for limnologists. Freshwater Biological Association, n. 36, 121 p., 1978.

Magurran, A. E. 1988. Ecological Diversity and its Measurement. Cambridge, University Press, London, $179 \mathrm{p}$.

MERRIT, R.; CUMMINS, K. An Introduction to the aquatic insects of North America. 2 ed. Kendall: Hunt Publishing, 1984.

MONTAG, L. F. A.; SMITH, W. S.; BARRELLA, W.; PETRERE Jr., M. As influências e as relações das matas ciliares nas comunidades de peixes do Estado de São Paulo. Revista Brasileira de Ecologia, São Paulo, v. 1, p. 76-80, 1997.

MORENO, P.; CALLISTO, M. Indicadores biológicos: a vida na lama. Ciência Hoje, vol. 36, n. 213, p. 68-71, 2005. 
MORETTI, M. S.; MORENO, P. Ecologia e funcionamento de ecossistemas aquáticos. Mini curso de Biologia, Universidade do Vale do Sapucaí, 2006.

PASSOS, M. J. Estrutura da vegetação arbórea e regeneração natural em remanescentes de mata ciliar no Rio Mogi/Guaçu, SP. 68f. 1998. Dissertação (Mestrado em Ciências Florestais). Escola Superior de Agricultura Luiz de Queiroz, Piracicaba, SP, 1998.

PÉREZ, G. A. R. Guía para el estudio de los macroinvertebrados acuáticos del Departamento de Antioquia. Bogotá: Fondo Fen Colômbia, Colciencias/Universidad de Antioquia, 1988.

RIGHI, G. 1984. Manual de identificação de invertebrados límnicos do Brasil. CNpq/Coordenação Editorial, 48p.

TACHET, H.; BOUNARD, M.; RICHOUX, P. Introduction á l'étude des Macroinvértebrés des eaux douces. Paris : C.R.D.P., 1987.

TER BRAAK, C.J.F.; ŠMILAUER, P. Canoco reference manual and CanoDraw for Windows user's guide: software for canonical community ordination. Microcomputer Power, Ithaca, NY, 2002.

TRIVINHO-STRIXINO, S.; STRIXINO. G. Larvas de Chironomidae (Diptera do Estado de São Paulo: guia de identificação de diagnose dos gêneros). São Carlos: Universidade Federal de São Carlos, 1995.

VALDERRAMA, J. C. The simultaneous analysis of total nitrogen and phosphorus in natural waters. Mar. Chem, 1981.

WARD, D.; HOLMES, N.; JOSÉ, P. The New Rivers \& Wildlife Handbook. RSPP, NRA e The Wildlife Trusts, Bedfordshire, 1995. 
WASHINGTON, H. G. Diversity, biotic and similiarity indices. A review with special relevance to aquatic ecosystems. Water Research, New York, v. 18, n.6, p. 653-694, 1984

WETZEL, R. G. Liminology. Washington Sauders College Publ., 1983.

WILHM, J. L.; DORRIS, T. C. Biological parameters for water quality criteria. BioScience, Washington, v 18, n.6, p. 447-481, 1968 